\title{
Expression of Transforming Growth Factor Alpha and Their Receptor (EGFR) in Thyroid Carcinoma and Benign Patients
}

\author{
Tahani Abduljabbar Nasser*, Kareem Hamed Ghali* \& Hameed Naeem Mousa** \\ *Department of Biology, College of Science, Wasit University \\ ** Department of Pathology and Forensic Medicine, College of Medicine, Thi - Qar \\ University \\ **hamed@utq.edu.iq
}

\begin{abstract}
The current study was included 178 patients, 111 patients out them were affected with thyroid cancer, 46 patients were benign neoplastic and 21 individuals were no cancers and benign as control group. The study was carried out in Laboratory of College of Science, University of Wasit in collaboration with AL-Hussein Teaching Hospital and Ibn al-Bitar Laboratory Specialist in Thi Qar Province, from January 2015 to April 2016. Immunohistochemical technique was used to determination the expression of TGF $\alpha$ and their receptor EGFR in all studied cases. The study aimed to estimation the expression of these proteins in thyroid carcinogenesis and the possibility of transforming the benign patients to thyroid cancer. The results showed overexpression in TGF $\alpha$ in thyroid cancer when compared with benign patients and control group with significant differences $(\mathrm{p}<0.05)$. Regarding EGFR, the results explained no significant differences when compared between three samples. Our results concluded there is a strong relationship between $\mathrm{TGF} \alpha$ overexpression and thyroid carcinogenesis.
\end{abstract}

Key words: Thyroid cancer, TGFa, EGFR and Immunohistochemistry.

\section{Introduction}




\section{Thi-Qar Medical Journal (TQMJ): Vol.(12), No.(2), 2016 \\ Email:utjmed@utq.edu.iq \\ Web Site: https://imed.utq.edu.iq}

Thyroid carcinoma is the most widespread type among endocrine malignancy (1). This cancer occurs in the thyroid gland, which it is importantly endocrine glands located at the lower part of the larynx, and responsible for the production of hormones that regulate the control: heart rate, blood pressure, body temperature, and basal metabolic rate (2). The occurrence of Thyroid cancer has increased significantly around the world over the last few decades (3). Which it is the fifth more commonly malignant tumor at women (4). And risk factors of thyroid cancer are very high in Arab countries (5). In Saudi Arabia, thyroid carcinoma was occupied the second ranked in females (6). While in Iraq it was occupies the 11th ranked among females (7). Thyroid carcinoma is more widespread in females at a rate of three times more than men, and is more predominant in the white persons than black persons, also common in Asian/Pacific Islander more than in other populations (8). According to histopathological characteristics, thyroid carcinoma has been split into two types: well differentiated, which include papillary and follicular carcinoma, and poorly differentiated, and this include medullary and anaplastic carcinoma (9). There are other categories of thyroid cancer but uncommon, such as: Hürthle cell carcinoma which is variants of follicular (10). Also there are mucoepidermoid carcinoma, thyroid lymphoma, squamous cell thyroid carcinoma and sarcoma of thyroid (11). The epidermal growth factor receptor EGFR is glycoprotein existing on the surface of some cells and composed of 1186 amino acid (12). Human EGFR gene is located on chromosome 7p12.3-p12.1 (13). EGFR is a member in the receptor tyrosine kinase family which members play a significant role in promoting cell growth, division, apoptosis and cell survival (14). The EGFR have sites allows it to attaching to other proteins, called ligands, in the outside of the cell and to receive signals that help the cell respond to the surrounding environment, and suitable together such as to locks and keys (15). EGFR is activated by binding of its own ligands, including epidermal growth factor (EGF) and transforming growth factor alpha (TGF $\alpha$ ), this kinase triggers diversely downstream signaling pathways and results in various biological responses, such as proliferation and differentiation (16).

Transforming growth factor alpha (TGF- $\alpha$ ): is a protein contain of 50 amino-acids polypeptide, the cytogenetic location of human TGF- $\alpha$ gene is: 2 p13.3, which is the short (p) arm of chromosome 2 at position 13.3 (17) TGF- $\alpha$ becomes effective when it is linked to receptors capable of protein kinase effectively of cellular signaling (18). 
TGF- $\alpha$ is a ligand for the EGF receptor, and because TGF- $\alpha$ is a member of the receptor tyrosine kinase family, so this gives them the similarity biological structural about $30 \%$, therefore TGF- $\alpha$ and EGF are linked to the same receptor (19). Loss of regulation of the TGF-alpha and its receptor EGFR could lead to many human diseases, most notably cancer (20).

In the recent period, the increased of thyroid cancer was affected by numerous factors such as genetic changes, growth agents, and physical factors such as radiation, so there are needed to helpful forecast factors to detect the biologic behavior, providing an initial help (21). A large number of biomarkers have been used in differential diagnosis of thyroid cancer, which include: TGF- $\alpha$, and EGFR have been translated in clinical applications that led to the emergence of a significant improvement in the preoperative diagnosis of thyroid cancer (22).

\section{Materials and methods}

\section{Patients and tissue samples}

The study was conducted in the laboratories of College of Science, University of Wasit and in collaboration with AL-Hussein Teaching Hospital, Thi Qar Province and Ibn al-Bitar National Laboratory in Thi Qar Province for the period from January 2014 to April 2016.One hundred and seventy-eight patients with diseases of the thyroid gland, which included three group of thyroid gland diseases 178 samples. first group is thyroid carcinoma 111 (62.36\%) patient's ,second group benign tumor account 46 (25.84\%) patient's and 21(11.80\%) other thyroid diseases (non-cancer or benign) the rate of the patient's age between 13 - 80 years. All patients were diagnosed and confirmed by specialized histopathlogistis. All clinical information about samples was taken. Histological samples were taken treated with formalin and embedded with paraffin wax postoperative.

\section{Immunohistochemistry (IHC):}

Serial tissue sections were cut 4- $5 \mu \mathrm{m}$ thick and positioned on positive charged slides. The slides were baked in $60^{\circ} \mathrm{C}$ oven over night. The tissue sections were deparaffinized; then the slides were dehydrated by graded ethanol concentration 
$(100 \%, 95 \%$, and $70 \%)$ and distal water. The slides were treated with citric buffer for 15 minute, and then washed in two changes of PBS solution for 5 minutes .Then slides were washed in distal water. The percentage of expression was calculated as 0 for negative, 1 for 1-25,2 for 26-50 ,and 3 for 51-100 staining cells. Intensity was calculated as 1 (weak), 2 (moderate) and 3 (strong)

\section{Ethical consent}

The study was submitted and approved by the College of Science, University of Wasit in collaboration with AL- Hussein Teaching Hospital and Ibn al-Bitar Laboratory $\begin{array}{llll}\text { Specialist } & \text { in } & \text { Thi } & \text { Parovince }\end{array}$

\section{Statistical analysis}

All the clinical, pathological, follow-up, expression data were computerized. Statistical analysis was performed using the SPSS for Windows (version 23.0). Fisher's exact test, chi-square test or likelihood ratio was used for categorical variables. Statistical significance level of the tests was taken at a p-value $<0.05$.

\section{Results}

\section{1-TGF $\alpha$ expression and intensity}

The results of immunohistochemical showed, that the expression of TGF $\alpha$ was positively in $76(68.47 \%)$ of thyroid carcinoma patients out of 111 cases, while in benign $20(43.48 \%)$ patients has appeared positive of 46 cases, as for the control group; $4(19.05 \%)$ cases are positive expression from 21 cases.

When thyroid carcinoma were compared with benign neoplasms showed there was highly significant differences between thyroid carcinoma patients and benign neoplasms to $\mathrm{TGF} \alpha$ expression $(\mathrm{P}=0.002)$ table $(1)$, as well as when comparing thyroid carcinoma with control group (table 2), the results showed that also there were highly significant differences between thyroid carcinoma patients and control group $(\mathrm{P}=0.000)$. Also benign patients and control group cases had appeared a significant differences $(\mathrm{P}=0.046)$ table $(3)$. 


\section{Thi-Qar Medical Journal (TQMJ): Vol.(12), No.(2), 2016 \\ Email:utjmed@utq.edu.iq \\ Web Site: https://imed.utq.edu.iq}

Regarding to the intensity evaluation of TGF- $\alpha$ expression in thyroid carcinoma patients revealed that 35 (31.53\%) patients were negative (score 0), while the intensity of expression was positive in $76(68.5 \%)$ of cases, which were distributed as follows: score $+1 ; 19(17.12 \%)$ case, score $+2 ; 43(38.74 \%)$ case and score $+3 ; 14(12.61 \%)$ of case. In benign neoplasms intensity of TGF- $\alpha$ expression was: $26(56.52 \%)$ case were negative (score 0$)$ and $20(43.48 \%)$ was positive divided as follows: $3(6.52 \%)$ cases were score $+1,15(32.61 \%)$ cases were score +2 and $2(4.35 \%)$ cases were score +3 . Either in control group the intensity of TGF- $\alpha$ expression was: 17 (80.95\%) negative (score 0$)$, while only $4(19.05 \%)$ cases were positive and distributed as: score $+1 ; 1$ $(4.76 \%)$ case, score $+2 ; 3(14.29 \%)$ case, and there was no any case in score +3 . When thyroid carcinoma were compared with benign neoplasms showed there was no significant differences in relation to intensity of TGF $\alpha$ expression $(\mathrm{P}>0.05)$, also there was no significant differences between thyroid carcinoma and control group $(\mathrm{P}>0.05)$. As well was the case for the relation to intensity of TGF $\alpha$ expression between benign neoplasms and control group which no statistical significance differences was found $(\mathrm{P}>0.05)$. Comparison of TGF expression intensity between thyroid carcinoma and benign patients and the control group are shown in Tables (1), (2) and (3).

\section{1- EGFR expression and intensity}

The result of EGFR expression was demonstrated positive expression at $56(50.45 \%)$ from 111 cases of thyroid carcinoma patients, and $15(32.61 \%)$ cases of benign patients are positive out of 46 case, while in control group 7 (33.33\%) cases were showed positive expression from 21 case.

By comparing EGFR expression between thyroid carcinoma patients and benign patients, the results demonstrated there was no statistically significant difference ( $p$ > 0.05), also no significant difference of expression between thyroid carcinoma patients and control group ( $p>0.05$ ), and this case also applies when comparing EGFR expression of benign patients and control group, there was no significant difference between expression ( $\mathrm{p}>0.05)$.

With respect to intensity assessment of EGFR expression in thyroid carcinoma patients detect that: $55(49.55 \%)$ cases were score $0,15(13.51 \%)$ cases with score +1 , $35(31.53 \%)$ cases with score +2 , and lowest percentage in $6(5.41 \%)$ cases were score +3 . As for intensity of benign patients were as follows: score $0 ; 31(67.39 \%)$ cases, score $+1 ; 5(10.87 \%)$ cases, score +2 ; 9 (19.57\%) cases, and only one case $(2.17 \%)$ in score +3 . In control group, $14(66.67 \%)$ cases were score $0,5(23.81 \%)$ 
cases with score $+1,2(9.52 \%)$ cases with score +2 , and score +3 ; with no any case $(0.00 \%)$.

In relation to intensity of EGFR expression there was no statistically significant difference between thyroid carcinoma and benign patients $(\mathrm{P}>0.05)$, as well as when the comparison between thyroid carcinoma and control group there was no significant differences $(\mathrm{P}>0.05)$, also is the situation between benign neoplasms and control group, no statistical significance differences was found $(\mathrm{P}>0.05)$.

Tables (4), (5) and (6) illustrate the Comparison of EGFR expression and intensity between thyroid carcinoma and benign neoplasms patients and the control group.

Table (1): TGF $\alpha$ Expression and Intensity in Thyroid Carcinoma compared with Benign Patients

\begin{tabular}{|c|c|c|c|c|c|c|c|}
\hline \multirow[t]{2}{*}{ Cases } & \multicolumn{2}{|c|}{ TGF $\alpha$ Expression } & \multicolumn{4}{|c|}{ TGF $\alpha$ Intensity } & \multirow{2}{*}{$\begin{array}{c}\text { Total } \\
\text { No. } \\
\%\end{array}$} \\
\hline & $\begin{array}{l}\text {-ve } \\
\text { No. } \\
\text { \% }\end{array}$ & $\begin{array}{c}+ \text { ve } \\
\text { No. } \\
\%\end{array}$ & $\begin{array}{c}0 \\
\text { No. } \\
\%\end{array}$ & $\begin{array}{c}1+ \\
\text { No. } \\
\%\end{array}$ & $\begin{array}{c}2+ \\
\text { No. } \\
\%\end{array}$ & $\begin{array}{c}3+ \\
\text { No. } \\
\%\end{array}$ & \\
\hline $\begin{array}{c}\text { Thyroid } \\
\text { Carcinoma } \\
\text { Patients }\end{array}$ & $\begin{array}{c}35 \\
31.53 \%\end{array}$ & $\begin{array}{c}76 \\
68.47 \%\end{array}$ & $\begin{array}{c}35 \\
31.53 \%\end{array}$ & $\begin{array}{c}19 \\
17.12 \%\end{array}$ & $\begin{array}{c}43 \\
38.74 \%\end{array}$ & $\begin{array}{c}14 \\
12.61 \%\end{array}$ & $\begin{array}{c}111 \\
100.0 \%\end{array}$ \\
\hline $\begin{array}{l}\text { Benign } \\
\text { Patients }\end{array}$ & $\begin{array}{c}26 \\
56.52 \%\end{array}$ & $\begin{array}{c}20 \\
43.48 \%\end{array}$ & $\begin{array}{c}26 \\
56.52 \%\end{array}$ & $\begin{array}{c}3 \\
6.52 \%\end{array}$ & $\begin{array}{c}15 \\
32.61 \%\end{array}$ & $\begin{array}{c}2 \\
4.35 \%\end{array}$ & $\begin{array}{c}46 \\
100.0 \%\end{array}$ \\
\hline \multirow[t]{2}{*}{ Total } & $\begin{array}{c}61 \\
38.85 \%\end{array}$ & $\begin{array}{c}96 \\
61.15 \%\end{array}$ & $\begin{array}{c}61 \\
38.85 \%\end{array}$ & $\begin{array}{c}22 \\
14.01 \%\end{array}$ & $\begin{array}{c}58 \\
36.94 \%\end{array}$ & $\begin{array}{c}16 \\
10.20 \%\end{array}$ & $\begin{array}{c}157 \\
100.0 \%\end{array}$ \\
\hline & \multicolumn{2}{|c|}{$P=0.002$} & \multicolumn{4}{|c|}{$P=0.378$} & \\
\hline
\end{tabular}

Table (2): TGFa Expression and Intensity in Thyroid Carcinoma compared with Control Group of Thyroid

\begin{tabular}{|c|c|c|c|c|c|c|c|}
\hline \multirow[t]{2}{*}{ Cases } & \multicolumn{2}{|c|}{ TGFa Expression } & \multicolumn{4}{|c|}{ TGFa Intensity } & \multirow{2}{*}{$\begin{array}{l}\text { Total } \\
\text { No. } \%\end{array}$} \\
\hline & $\begin{array}{c}\text {-ve } \\
\text { No. } \\
\%\end{array}$ & $\begin{array}{l}+ \text { ve } \\
\text { No. } \\
\%\end{array}$ & $\begin{array}{c}\text { 0 } \\
\text { No. } \\
\%\end{array}$ & $\begin{array}{c}1+ \\
\text { No. } \\
\%\end{array}$ & $\begin{array}{l}+r \\
\text { No. } \\
\%\end{array}$ & $\begin{array}{c}+r \\
\text { No. } \\
\%\end{array}$ & \\
\hline $\begin{array}{l}\text { Thyroid } \\
\text { Carcinoma } \\
\text { Patients }\end{array}$ & $\begin{array}{c}35 \\
31.53 \%\end{array}$ & $\begin{array}{c}76 \\
68.47 \%\end{array}$ & $\begin{array}{c}35 \\
31.53 \%\end{array}$ & $\begin{array}{c}19 \\
17.12 \%\end{array}$ & $\begin{array}{c}43 \\
38.74 \%\end{array}$ & $\begin{array}{c}14 \\
12.61 \%\end{array}$ & $\begin{array}{c}111 \\
100.0 \%\end{array}$ \\
\hline $\begin{array}{l}\text { Control } \\
\text { Group } \\
\text { Patient }\end{array}$ & $\begin{array}{c}17 \\
80.95 \%\end{array}$ & $\begin{array}{c}4 \\
19.05 \%\end{array}$ & $\begin{array}{c}17 \\
80.95 \%\end{array}$ & $\begin{array}{c}1 \\
4.76 \%\end{array}$ & $\begin{array}{c}3 \\
14.29 \%\end{array}$ & $\begin{array}{c}0 \\
0.00 \%\end{array}$ & $\begin{array}{c}21 \\
100.0 \%\end{array}$ \\
\hline
\end{tabular}


Thi-Qar Medical Journal (TQMJ): Vol.(12), No.(2), 2016

Email:utjmed@utq.edu.iq

Web Site: https://imed.utq.edu.iq

\begin{tabular}{|c|c|c|c|c|c|c|c|}
\hline Total & 52 & 80 & 52 & 20 & 46 & 14 & 132 \\
& $39.39 \%$ & $60.61 \%$ & $39.39 \%$ & $15.15 \%$ & $34.85 \%$ & $10.61 \%$ & $100.0 \%$ \\
\hline & \multicolumn{5}{|c|}{$\mathbf{P}=\mathbf{0 . 0 0 0}=\mathbf{1 . 0 0 0}$} \\
\hline
\end{tabular}

Table (3): TGFa Expression and Intensity in Benign Patients and Control Group of Thyroid

\begin{tabular}{|c|c|c|c|c|c|c|c|}
\hline \multirow[t]{2}{*}{ Cases } & \multicolumn{2}{|c|}{ TGFa Expression } & \multicolumn{4}{|c|}{ TGF $\alpha$ Intensity } & \multirow{2}{*}{$\begin{array}{c}\text { Total } \\
\text { No. } \\
\%\end{array}$} \\
\hline & $\begin{array}{l}\text {-ve } \\
\text { No. } \\
\%\end{array}$ & $\begin{array}{l}\text { +ve } \\
\text { No. } \\
\%\end{array}$ & $\begin{array}{c}\text { 0 } \\
\text { No. } \\
\%\end{array}$ & $\begin{array}{l}+1 \\
\text { No. } \\
\%\end{array}$ & $\begin{array}{l}+r \\
\text { No. } \\
\%\end{array}$ & $\begin{array}{c}+r \\
\text { No. } \\
\%\end{array}$ & \\
\hline $\begin{array}{c}\text { Benign } \\
\text { Neoplasms } \\
\text { Patient }\end{array}$ & $\begin{array}{c}26 \\
56.52 \%\end{array}$ & $\begin{array}{c}20 \\
43.48 \%\end{array}$ & $\begin{array}{c}26 \\
56.52 \%\end{array}$ & $\begin{array}{c}3 \\
6.52 \%\end{array}$ & $\begin{array}{c}15 \\
32.61 \%\end{array}$ & $\begin{array}{c}2 \\
4.35 \%\end{array}$ & $\begin{array}{c}46 \\
100.0 \%\end{array}$ \\
\hline $\begin{array}{l}\text { Control } \\
\text { Group } \\
\text { Patient }\end{array}$ & $\begin{array}{c}17 \\
80.95 \%\end{array}$ & $\begin{array}{c}4 \\
19.05 \%\end{array}$ & $\begin{array}{c}17 \\
80.95 \%\end{array}$ & $\begin{array}{c}1 \\
4.76 \%\end{array}$ & $\begin{array}{c}3 \\
14.29 \%\end{array}$ & $\begin{array}{c}0 \\
0.00 \%\end{array}$ & $\begin{array}{c}21 \\
100.0 \%\end{array}$ \\
\hline \multirow[t]{2}{*}{ Total } & $\begin{array}{c}43 \\
64.18 \%\end{array}$ & $\begin{array}{c}24 \\
35.82 \%\end{array}$ & $\begin{array}{c}43 \\
64.18 \%\end{array}$ & $\begin{array}{c}4 \\
5.97 \%\end{array}$ & $\begin{array}{c}18 \\
26.87 \%\end{array}$ & $\begin{array}{c}2 \\
2.98 \%\end{array}$ & $\begin{array}{c}67 \\
100.0 \%\end{array}$ \\
\hline & \multicolumn{2}{|c|}{$P=0.046$} & \multicolumn{4}{|c|}{$P=1.000$} & \\
\hline
\end{tabular}

Table (4): EGFR Expression and Intensity in Thyroid Carcinoma compared with Benign Patients

\begin{tabular}{|c|c|c|c|c|c|c|c|}
\hline \multirow[t]{2}{*}{ Cases } & \multicolumn{2}{|c|}{$\begin{array}{c}\text { EGFR } \\
\text { Expression }\end{array}$} & \multicolumn{4}{|c|}{ EGFR Intensity } & \multirow{2}{*}{$\begin{array}{c}\text { Total } \\
\text { No. } \\
\%\end{array}$} \\
\hline & $\begin{array}{l}\text {-ve } \\
\text { No. } \\
\%\end{array}$ & $\begin{array}{c}\text { +ve } \\
\text { No. } \\
\%\end{array}$ & $\begin{array}{c}\text { 0 } \\
\text { No. } \\
\%\end{array}$ & $\begin{array}{c}1+ \\
\text { No. } \\
\%\end{array}$ & $\begin{array}{r}2+ \\
\text { No. } \\
\%\end{array}$ & $\begin{array}{c}3+ \\
\text { No. } \\
\%\end{array}$ & \\
\hline $\begin{array}{l}\text { Thyroid } \\
\text { Carcinoma } \\
\text { Patients }\end{array}$ & $\begin{array}{c}55 \\
49.55 \%\end{array}$ & $\begin{array}{c}56 \\
50.45 \%\end{array}$ & $\begin{array}{c}55 \\
49.55 \%\end{array}$ & $\begin{array}{c}15 \\
13.51 \%\end{array}$ & $\begin{array}{c}35 \\
31.53 \%\end{array}$ & $\begin{array}{c}6 \\
5.41 \%\end{array}$ & $\begin{array}{c}111 \\
100.0 \%\end{array}$ \\
\hline $\begin{array}{l}\text { Benign } \\
\text { Patients }\end{array}$ & $\begin{array}{c}31 \\
67.39 \%\end{array}$ & $\begin{array}{c}15 \\
32.61 \%\end{array}$ & $\begin{array}{c}31 \\
67.39 \%\end{array}$ & $\begin{array}{c}5 \\
10.87 \%\end{array}$ & $\begin{array}{c}9 \\
19.57 \%\end{array}$ & $\begin{array}{c}1 \\
2.17 \%\end{array}$ & $\begin{array}{c}46 \\
100.0 \%\end{array}$ \\
\hline \multirow[t]{2}{*}{ Total } & $\begin{array}{c}86 \\
54.78 \%\end{array}$ & $\begin{array}{c}71 \\
45.22 \%\end{array}$ & $\begin{array}{c}86 \\
54.78 \%\end{array}$ & $\begin{array}{c}20 \\
12.74 \%\end{array}$ & $\begin{array}{c}44 \\
28.02 \%\end{array}$ & $\begin{array}{c}7 \\
4.46 \%\end{array}$ & $\begin{array}{c}157 \\
100.0 \%\end{array}$ \\
\hline & \multicolumn{2}{|c|}{$P=0.3$} & \multicolumn{4}{|c|}{$P=0.539$} & \\
\hline
\end{tabular}


Table (5): EGFR Expression and Intensity in Thyroid Carcinoma compared with Control Group of Thyroid Patients

\begin{tabular}{|c|c|c|c|c|c|c|c|}
\hline \multirow[t]{2}{*}{ Cases } & \multicolumn{2}{|c|}{ EGFR Expression } & \multicolumn{4}{|c|}{ EGFR Intensity } & \multirow{2}{*}{$\begin{array}{c}\text { Total } \\
\text { No. } \\
\%\end{array}$} \\
\hline & $\begin{array}{l}\text {-ve } \\
\text { No. } \\
\text { \% }\end{array}$ & $\begin{array}{l}\text { +ve } \\
\text { No. } \\
\%\end{array}$ & $\begin{array}{c}\text { 0 } \\
\text { No. } \\
\%\end{array}$ & $\begin{array}{c}1+ \\
\text { No. } \\
\%\end{array}$ & $\begin{array}{l}2+ \\
\text { No. } \\
\%\end{array}$ & $\begin{array}{c}3+ \\
\text { No. } \\
\%\end{array}$ & \\
\hline $\begin{array}{l}\text { Thyroid } \\
\text { Carcinoma } \\
\text { Patients }\end{array}$ & $\begin{array}{c}55 \\
49.55 \%\end{array}$ & $\begin{array}{c}56 \\
50.45 \%\end{array}$ & $\begin{array}{c}55 \\
49.55 \%\end{array}$ & $\begin{array}{c}15 \\
13.51 \%\end{array}$ & $\begin{array}{c}35 \\
31.53 \%\end{array}$ & $\begin{array}{c}6 \\
5.41 \%\end{array}$ & $\begin{array}{c}111 \\
100.0 \%\end{array}$ \\
\hline $\begin{array}{l}\text { Control } \\
\text { Group } \\
\text { Patient }\end{array}$ & $\begin{array}{c}14 \\
66.67 \%\end{array}$ & $\begin{array}{c}7 \\
33.33 \%\end{array}$ & $\begin{array}{c}14 \\
66.67 \%\end{array}$ & $\begin{array}{c}5 \\
23.81 \%\end{array}$ & $\begin{array}{c}2 \\
9.52 \%\end{array}$ & $\begin{array}{c}0 \\
0.00 \%\end{array}$ & $\begin{array}{c}21 \\
100.0 \%\end{array}$ \\
\hline \multirow[t]{2}{*}{ Total } & $\begin{array}{c}69 \\
52.27 \%\end{array}$ & $\begin{array}{c}63 \\
47.73 \%\end{array}$ & $\begin{array}{c}69 \\
52.27 \%\end{array}$ & $\begin{array}{c}20 \\
15.15 \%\end{array}$ & $\begin{array}{c}37 \\
28.03 \%\end{array}$ & $\begin{array}{c}6 \\
4.55 \%\end{array}$ & $\begin{array}{c}132 \\
100.0 \%\end{array}$ \\
\hline & \multicolumn{2}{|c|}{$P=0.069$} & \multicolumn{4}{|c|}{$P=0.079$} & \\
\hline
\end{tabular}

Table (6): EGFR expression and intensity in benign patients and control group of thyroid patients

\begin{tabular}{|c|c|c|c|c|c|c|c|}
\hline \multirow[t]{2}{*}{ Cases } & \multicolumn{2}{|c|}{ EGFR Expression } & \multicolumn{4}{|c|}{ EGFR Intensity } & \multirow{2}{*}{$\begin{array}{c}\text { Total } \\
\text { No. } \\
\%\end{array}$} \\
\hline & $\begin{array}{l}\text {-ve } \\
\text { No. } \\
\%\end{array}$ & $\begin{array}{l}+ \text { ve } \\
\text { No. } \\
\%\end{array}$ & $\begin{array}{c}\text { 0 } \\
\text { No. } \\
\%\end{array}$ & $\begin{array}{r}1+ \\
\text { No. } \\
\%\end{array}$ & $\begin{array}{r}2+ \\
\text { No. } \\
\%\end{array}$ & $\begin{array}{r}3+ \\
\text { No. } \\
\%\end{array}$ & \\
\hline $\begin{array}{l}\text { Benign } \\
\text { Patients }\end{array}$ & $\begin{array}{c}31 \\
67.39 \%\end{array}$ & $\begin{array}{c}15 \\
32.61 \%\end{array}$ & $\begin{array}{c}31 \\
67.39 \%\end{array}$ & $\begin{array}{c}5 \\
10.87 \%\end{array}$ & $\begin{array}{c}9 \\
19.57 \%\end{array}$ & $\begin{array}{c}1 \\
2.17 \%\end{array}$ & $\begin{array}{c}46 \\
100.0 \%\end{array}$ \\
\hline $\begin{array}{l}\text { Control } \\
\text { Group } \\
\text { Patient }\end{array}$ & $\begin{array}{c}14 \\
66.67 \%\end{array}$ & $\begin{array}{c}7 \\
33.33 \%\end{array}$ & $\begin{array}{c}14 \\
66.67 \%\end{array}$ & $\begin{array}{c}5 \\
23.81 \%\end{array}$ & $\begin{array}{c}2 \\
9.52 \%\end{array}$ & $\begin{array}{c}0 \\
0.00 \%\end{array}$ & $\begin{array}{c}21 \\
100.0 \%\end{array}$ \\
\hline \multirow[t]{2}{*}{ Total } & $\begin{array}{c}45 \\
67.16 \%\end{array}$ & $\begin{array}{c}22 \\
32.84 \%\end{array}$ & $\begin{array}{c}45 \\
67.16 \%\end{array}$ & $\begin{array}{c}10 \\
14.93 \%\end{array}$ & $\begin{array}{c}11 \\
16.42 \%\end{array}$ & $\begin{array}{c}1 \\
1.49 \%\end{array}$ & $\begin{array}{c}67 \\
100.0 \%\end{array}$ \\
\hline & \multicolumn{2}{|c|}{$P=0.219$} & \multicolumn{4}{|c|}{$P=0.235$} & \\
\hline
\end{tabular}




\section{Discussion}

This study has shown that there is a high significance difference of TGF $\alpha$ expression between thyroid carcinoma and benign patients $(\mathrm{P}<0.05)$, Also there was a highly significance difference of $\mathrm{TGF} \alpha$ expression between thyroid carcinoma and noncancerous control group, where the TGF- $\alpha$ overexpression was positive in $(68.47 \%)$ of thyroid carcinoma patients, $(43.48 \%)$ in benign patients and $(19.05 \%)$ in noncancerous control group, this is compatible with results of Lam et al.(23) and Lau (24) .Overexpression of TGF $\alpha$ has been observed in a variety of human cancers, including hepatocellular cancer, a study done by Daveau, Scotte et al. (25), salivary duct cancer a study by Fan, Melhem et al. (26) and gastric cancer study by Konturek et al. (27). On the other hand there was no significant correlation of EGFR expression between thyroid carcinoma and benign patients, as well, there was no significant difference of EGFR expression between thyroid carcinoma and noncancerous control group, although the percentage of EGFR overexpression was positively in $(50.45 \%)$ of thyroid carcinoma and this is identical to the Lei Gong study (28), but the ratio was only $(32.61 \%)$ in benign neoplasms patients and (33.33\%) in noncancerous control group. In a study by Lam et al.(23), they found that was a high level of TGF- $\alpha$ expression was noted in $77 \%$ (55 from 71) of thyroid cancers, and also a high level of EGFR expression was observed in 54\% (38 from 71) of thyroid cancers .Additionally, in this study we compared TGF $\alpha$ and EGFR expression between benign neoplasms and noncancerous control group, whereas most of other studies were compared only among thyroid carcinoma patients with benign neoplasms or with noncancerous control group, in the current study we have noted there was significance difference of TGF $\alpha$ expression $(\mathrm{P}<0.05) \quad$ between benign patients and noncancerous control group, while there was no significant correlation of EGFR expression among benign neoplasms and noncancerous control group.In the study conducted by Lau (24), 59 patients of papillary thyroid carcinoma and 10 benign thyroid neoplasm was taken, and the results were all cases of the papillary thyroid carcinoma positively by TGF $\alpha$ expression compared with only $20 \%$ in the benign thyroid neoplasm, and from 59 samples, $39(66 \%)$ were higher expression and 20 (34\%) were lower expression, as for the benign thyroid neoplasms all positive samples were classified as a lower expression. Moreover, there was no significant difference of TGF $\alpha$ intensity between thyroid carcinoma and benign neoplasms patients $(\mathrm{P}>0.05)$, the same applies to TGF $\alpha$ intensity between thyroid carcinoma and noncancerous control group were there no significant difference 


\section{Email:utjmed@utq.edu.iq}

and this is consistent with results by Lau (24). Also there was a no significance correlation of the EGFR intensity between thyroid carcinoma and benign neoplasms patients, the same result for the EGFR intensity between thyroid carcinoma and noncancerous control group there was no significant correlation. Our results concluded there is a strong relationship between TGF $\alpha$ expression and thyroid cancer.

\section{References}

1- Nguyen, Q. T.; Lee, E. J.; Huang, M. G.; Park, Y. I.; Khullar, A. and Plodkowsk, R. A. (2015). Diagnosis and treatment of patients with thyroid cancer, American health \& drug benefits, 8 (1): 30-38.

2- Khatawkar, A. V. and Awati, S. M. (2015). Thyroid gland - historical, aspects, embryology anatomy and physiology, International Archives of Integrated Medicine, 2 (9).

3- Sipos, J. A. and Mazzaferri, E. L. (2010). Thyroid cancer epidemiology and prognostic variables, Clinical Oncology, 22 (6): 395-404.

4- Jemal, A.; Siegel, R.; Xu, J. and Ward, E. (2010). Cancer statistics, 2010, CA: a cancer journal for clinicians, 60 (5): 277 300.

5- Al Shahrani, A. S.; El-Metwally, A.; AlSurimi, K.; Bin-Salih, S.; Saleh, Y.; AlShehri, A. and Ali, A. (2016). The epidemiology of thyroid diseases in the
Web Site: https://imed.utq.edu.iq

Arab world: a systematic review, Journal of Public Health and Epidemiology, 8(2): $17-26$.

6- Hussain, F.; Iqbal, S.; Mehmood, A.; Bazarbashi, S.; El-Hassan, T. and Chaudhri, N. (2013). Incidence of thyroid cancer in the Kingdom of Saudi Arabia, 2000-2010, Hematology/Oncology and Stem Cell Therapy, 6 (2): 58-64.

7- Ferlay, J.; Soerjomataram, I.; Ervik, M.; Dikshit, R.; Eser, S.; Mathers, C.; Rebelo, M.; Parkin, D. M.; Forman, D. and Bray, F. (2015). Cancer incidence and mortality worldwide: Sources, methods and major patterns in GLOBOCAN 2012, International Journal of Cancer, 136 (5): 359-386.

8- National Cancer Institute (U.S.) (2015). SEER, Surveillance, Epidemiology, and End Results: thyroid cancer, U.S. Department of Health and Human Services, National Institutes of Health, National Cancer Institute.

9- Shrivastava, J. P.; Mangal, K. S.; Woike, P.; Marskole, P. and Gaur, R. (2016). Role of FNAC in diagnosing thyroid neoplasms - a retrospective study, IOSR Journal of Dental and Medical Sciences, 15 (1): 13-16.

10- Trigo, J. M.; Capdevila, J.; Grande, E.; Grau, J. and Lianes, P. (2014). Thyroid cancer: SEOM clinical guidelines, Clinical and Translational Oncology, 16 (12): 1035-1042.

11-Mazzaferri, E. L.; Harmer, C.; Mallick, U. K. and Taylor, P. K. (2006). Practical 


\section{Email:utjmed@utq.edu.iq}

management of thyroid cancer: a multidisciplinary approach, SpringerVerlag, London.

12- Herbst, R. S. (2004). Review of epidermal growth factor receptor biology, International Journal of Radiation Oncology, Biology, Physics: Supplement, 59 (2): 21-26.

13- Udart, Martin; U., Jochen; K., Gertraud M. and Peter, R. U. (2001). Chromosome 7 Aneusomy. A marker for metastatic melanoma: expression of the epidermal growth factor receptor gene and chromosome 7 Aneusomy in Nevi, primary malignant melanomas and metastases, Nature Publishing Group, 3 (3): 245-254.

14- Robinson D. R.; Wu, Y. and Lin, S. (2000). The protein tyrosine kinase family of the human genome, Oncogene, 19(49): 5548-5557.

15- Normanno, N.; De-Luca, A.; Bianco, C.; Strizzi, L.; Mancino, M.; Maiello, M. R.; Carotenuto, A.; De Feo, G.; Caponigro, F. and S., David S. (2006). Epidermal growth factor receptor (EGFR) signaling in cancer, Gene, 366(1): 2-16.

16-Bogdan, S. and Klambt, C. (2001). Epidermal growth factor receptor signaling, Current biology, 11(8): 292295.

17- Rosenbloom, K. R.; Armstrong, J.; Barber, G. P.; Casper, J. and Clawson, H. (2015). The UCSC genome browser database: 2015 update, Nucleic acids research, 43: 670-681.
Web Site: https://imed.utq.edu.iq

18- Wieduwilt, M. J. and Moasser, M. M. (2008). The epidermal growth factor receptor family: Biology driving targeted therapeutics, Cellular and molecular life sciences, 65 (10): 1566-1584

19-Acton, Q. A. (2013). Gastrointestinal hormone receptors: advances in research and application, Atlanta, Georgia.

20- Jameson, J. L.; DeGroot, L. J.; De Kretser, D. M.; Giudice, L.; Grossman, A.; Melmed, S.; Potts, J. T. and Weir, G. C. (2016). Endocrinology: adult \& pediatric, 7th edition, Elsevier/Saunders Philadelphia, PA.

21-Croyle, M.; Akeno, N.; Knauf, J. A.; Fabbro, D.; Chen, X.; Baumgartner, J. E.; Lane, H. A. and Fagin, J. A. (2008). RET/PTC-induced cell growth is mediated in part by epidermal growth factor receptor (EGFR) activation: evidence for molecular and functional interactions between RET and EGFR, Cancer Research, 68(11): 4183-4191.

22-Sethi, K.; Sarkar, S.; Das, S.; Mohanty, B. and Mandal, M. (2010). Biomarkers for the diagnosis of thyroid cancer, Journal of Experimental Therapeutics \& Oncology, 8 (4): 341-52.

23-Lam, A. K.; Lau, K. K.; Gopalan, V.; Luk, J. and Lo, C. Y. (2011). Quantitative analysis of the expression of transformation growth factor alpha (TGF$\alpha$ ) and epidermal growth factor receptor (EGFR) in papillary thyroid carcinoma: clinicopathological relevance, Pathology, 43 (1): 40-47. 


\section{Email:utjmed@utq.edu.iq}

24- Lau, K. P. (2007). Clinicopathological Roles of Transforming Growth Factor Alpha (TGF $\alpha)$ in Papillary Thyroid Carcinoma, Hong Kong University Theses Online.

25- Daveau, M.; Scotte, M.; FrançOis, A.; Coulouarn, C.; Ros, G.; Tallet, Y.; Hiron, M.; Hellot, M.-F. and Salier, J. P. (2003). Hepatocyte growth factor, transforming

growth factor alpha, and their receptors as combined markers of prognosis in hepatocellular carcinoma, Molecular Carcinogenesis, 36 (3): 130-141.

26- Fan, C. Y.; Melhem, M. F.; Hosal, A. s.; Grandis, J. r. and Barnes, E. I. (2001). Expression of androgen receptor, epidermal growth factor receptor, and transforming growth factor alpha in
Web Site: https://imed.utq.edu.iq

salivary duct carcinoma, Archives of Otolaryngology Head \& Neck Surgery, 127 (9): 1075-1079.

27-Konturek， P. C.; Konturek，S. J.; Sulekova, Z.; Meixner, H.; Bielanski, W.; Starzynska, T.; Karczewska, E.; Marlicz, K.; Stachura, J. and Hahn, E. g. (2001).

28-Expression of hepatocyte growth factor, transforming growth factor alpha, apoptosis related proteins Bax and Bcl-2, and gastrin in human gastric cancer, Alimentary Pharmacology \& Therapeutics, 15 (7): 989-999.

29-Gong, L.; Chen, P.; Liu, X.; Han, Y.; Zhou, Y.; Zhang, W.; Li, H.; Li, C. and Xie, J. (2012). Expressions of D2-40, CK19, galectin-3, VEGF and EGFR in papillary thyroid carcinoma, Gland Surg, 1 (1): 25-32. 


\section{تعبير البروتين TGF- ألفا ومستقبله EGFR في مرضى السرطان والاورام الحميدة للغذة الدرقية}

حميا نعيم موسى

كريم حمد غالي

تهاني عبد الجبار ناص

الخلاصة :

تضمنت الدراسة الحالية 178 مريضا ، 111 مريضا مصابا بسرطان الغدة الدرقية و 26 مريضا مصابا بالاورام الحميدة للغدة الدرقية و 21 دريضا مصابا بأمراض اخرى ( غير سرطانية و غير حميدة ) كمجمو عة سيطرة ـ انجزت الدر اسة للفترة من كانون الثاني 2015 الى نيسان 2016 في مختبرات كلية العلوم / جامعة واسط بالتعاون مع مستشفى الحسين التعليمي ومختبر ابن البيطار التخصصي في محافظة ذي قار ـ استخدمت تقنية التصبيغ المناعي النسيجي الكيميائي لتحديد تعبير البروتينات TGFa و EGFR في جميع العينات المدروسة ـ تهدف الدر اسة الى تحديد التعبير الجيني للبروتينات المذكورة في العينات قيد الدراسة لتقييم دور هذه البروتينات في الاصابة بسرطان الغدة الدرقية وامكانية تحول مرضى الاورام الحميدة مستقبلا الى مصابين بسرطان الدرقية ـ اظهرت النتائج زيادة في تعبير TGFa في مرضى سرطان الدرقية مقارنة بمرضى اورام الدرقية الحميدة و مع عينة السيطرة وبفارق معنوي (P 0.05 ( كذلك نفس الفرق المعنوي ظهر عند المقارنة بين عينة الاورام الحميدة وعينة السيطرة ـ اما بخصوص البروتين EGFR فقد اوضحت النتائج عدم وجود اي فرق معنوي عند المقارنة بين عينات مرضى سرطان الدرقية ومرضى الاورام الحميدة للارقية وعينة السيطرة وكذلك عند المقارنة بين عينتي الاورام الحميدة والسيطرة ـ تسنتج الدراسة الحالية وجود علاقة قوية بين البروتين TGFa و الاصنابة بسرطان الدرقية .

الكلمات المفتاحية : سرطان الغدة الدرقية، التصبيغ المناعي النسيجي , TGFa \& EGFR الكيميائي . 